\title{
The study of phase transition in systems with the competing interactions in a magnetic field by computer modeling
}

\author{
S. V. Belim \\ sbelim@mail.ru \\ Omsk State Technical University, 11 Mira Av., Omsk, 644050, Russia \\ Siberian State Automobile and Highway University, 5 Mira Av., Omsk, 644080, Russia
}

In this article, spin systems with competing interactions in an external magnetic field are investigated. The Ising model with ferromagnetic short-range forces and long-range antiferromagnetic forces is considered. Long-range forces decrease according to the power law. Long-range interaction is characterized by the rate of decrease with distance and relative intensity. The Metropolis algorithm is used for computer modeling. Two order parameters for ferromagnetic and antiferromagnetic ordering are used. The theory of finite size scaling is used. The transition temperature was defined based on Binder's cumulants for each of the order parameters. The phase diagram for the system is constructed at various values of the longrange interaction parameters. As the temperature decreases, the system can transfer from the paramagnetic phase into the ferromagnetic and antiferromagnetic ones. The type of the low-temperature phase depends on the intensity of long-range forces and does not depend on the rate of decrease of the interaction with distance. There is a boundary value of the intensity of long-range forces, below which the ferromagnetic phase is implemented, and above it is the antiferromagnetic phase. The change in the phase diagram under the influence of an external magnetic field is investigated. In an external magnetic field, the ferromagnetic phase transition becomes spread. The temperature of the antiferromagnetic phase transition in an external field decreases according to the square law. The boundary value of the intensity of long-range forces linearly grows with increasing the intensity of the external magnetic field. An increase in the boundary value in a magnetic field leads to a change in the type of the low-temperature phase. This effect is shown as a phase transition from the antiferromagnetic to the ferromagnetic phase in the external magnetic field. A comparison with the data of real experiments is carried out.

Keywords: competing interactions, ferromagnetics, antiferromagnetics, phase transition.

УДК: 537.622 .5

\section{Исследование фазовых переходов в системах с конкурирующими взаимодействиями в магнитном поле методом компьютерного моделирования}

\footnotetext{
Белим С. В.

Омский государственный технический университет, пр. Мира, 11, Омск, 644050, Россия

Сибирский государственный автомобильно-дорожный университет (СибАДИ), пр. Мира, 5, Омск, 644080, Россия

В статье проведено исследование спиновых систем с конкурирующими взаимодействиями во внешнем магнитном поле. Рассмотрена модель Изинга с ферромагнитными близкодействующими силами и дальнодействующими антиферромагнитными силами. Дальнодействующие силы убывают по степенному закону. Дальнодействующее взаимодействие характеризуется скоростью убывания с расстоянием и относительной интенсивностью. Для компьютерного моделирования использован алгоритм Метрополиса. Введены два параметра порядка для ферромагнитного и антиферромагнитного упорядочивания. Использована теория конечноразмерного скейлинга. Температура фазового перехода определялась на основе куммулянтов Биндера для каждого из параметров порядка. Построена фазовая диаграмма системы при различных значениях параметров дальнодействия. Показано, что при понижении температуры система может переходить из парамагнитной фазы как в ферромагнитную, так и антиферромагнитную фазу. Вид низкотемпературной фазы зависит от интенсивности дальнодействующих сил и не зависит от скорости убывания взаимодействия с расстоянием. Существует граничное значение интенсивности дальнодействующих
} 
сил, ниже которого реализуется ферромагнитная фаза, а выше - антиферромагнитная. Исследовано изменение фазовой диаграммы под действием внешнего магнитного поля. Во внешнем магнитном поле ферромагнитный фазовый переход становится размытым. Температура антиферромагнитного фазового перехода во внешнем поле убывает по квадратичному закону. Граничное значение интенсивности дальнодействующих сил линейно растет с увеличением напряженности внешнего магнитного поля. Изменение граничного значения под действием магнитного поля приводит к изменению типа низкотемпературной фазы. Этот эффект проявляется как фазовый переход из антиферромагнитной в ферромагнитную фазу под действием внешнего магнитного поля. Проведено сравнение с данными реальных экспериментов.

Ключевые слова: конкурирующие взаимодействия, антиферромагнетики, ферромагнетики, фазовые переходы.

\section{1. Введение}

Наличие конкурирующих ферромагнитных и антиферромагнитных взаимодействий наблюдается в ряде материалов. Так в работе [1] экспериментально, а в [2] на основе расчетов из первых принципов показано, что в перовскитах $\mathrm{Sr}_{2} \mathrm{NiIrO}_{6}$ и $\mathrm{Sr}_{2} \mathrm{ZnIrO}_{6}$ взаимодействие между спинами описывается близкодействующими ферромагнитными силами и дальнодействующими антиферромагнитными силами. Кроме этого сосуществование ферромагнитных и антиферромагнитных сил наблюдалось экспериментально и в дальнейшем было подтверждено в рамках теории функционала плотности для сплавов $\mathrm{Ni}_{2} \mathrm{MnGa}$ [3].

Системы с конкурирующими взаимодействиями ранее были исследованы в рамках теоретикополевого подхода $[4,5,6]$ и методами компьютерного моделирования $[7,8,9]$. Исследования показали, что характер фазового перехода зависит от относительной интенсивности взаимодействий. Существует пороговое значение, выше которого более слабые антиферромагнитные взаимодействия доминируют над ферромагнитными за счет влияния спинов следующих за ближайшими соседями. В результате на фазовой диаграмме системы присутствует трикритическая точка, обусловленная существованием трех фаз - парамагнитной, антиферромагнитной и ферромагнитной.

Из экспериментальных исследований [10,11] и результатов компьютерного моделирования $[12,13,14]$ известно, что наличие внешнего магнитного поля приводит к подавлению антиферромагнитной фазы. В частности, температура Нееля убывает при росте напряженности внешнего магнитного поля по квадратичному закону. В связи с этим можно ожидать существенного влияния внешнего магнитного поля на характер фазовых переходов с конкурирующими ферромагнитным и антиферромагнитным взаимодействиями. Внешнее магнитное поле будет способствовать ферромагнитному упорядочиванию спинов и подавлять антиферромагнитное. Вследствие этого можно ожидать увеличения порогового значения относительной интенсивности дальнодействующих антиферромагнитных сил, при котором реализуется антиферромагнитная фаза. Для реальных веществ с конкурирующими взаимодействиями этот эффект может проявляться как фазовый переход из анти- ферромагнитной фазы в ферромагнитную под действием внешнего магнитного поля.

Целью данной статьи является исследование фазовых переходов в ферромагнитной модели Изинга с дальнодействующими антиферромагнитными силами во внешнем магнитном поле методом компьютерного моделирования.

\section{2. Описание системы}

Рассмотрим систему, в которой действует обменное ферромагнитное взаимодействие и дальнодействующее антиферромагнитное взаимодействие. Причем антиферромагнитные силы убывают по степенному закону. Свободная энергия такой системы может быть записана в следующем виде:

$$
F=J \sum S_{i} S_{j}-\frac{b J}{r^{-D-\sigma}} \sum S_{i} S_{j}+\mu H \sum S_{i},
$$

где $S_{i}-$ значения спина в $i$-ом узле $(+1 / 2$ или $-1 / 2)$, $J$ - обменный интеграл ферромагнитного близкодействующего взаимодействия, $b-$ относительная интенсивность дальнодействующего антиферромагнитного взаимодействия, $r-$ расстояние между спинами, $D-$ размерность пространства, $\sigma-$ константа дальнодействия, $\mu$ - магнетон Бора, $h-$ напряженность магнитного поля. В первом слагаемом суммирование выполняется только по парам ближайших соседей, во втором слагаемом учитываются не только ближайшие соседи, но и следующие за ближайшими соседями спины.

При компьютерном моделировании удобнее работать с безразмерными величинами. Введем приведенные свободную энергию и напряженность магнитного поля:

$$
f=F / J, h=\mu H / J \text {. }
$$

Тогда свободная энергия запишется в следующем виде:

$$
f=\sum S_{i} S_{j}-\frac{b}{r^{-D-\sigma}} \sum S_{i} S_{j}+h \sum S_{i}
$$

Приведенная температура $T$ такой системы также будет выражаться через величину обменного интеграла $T=k t / J$, где $t-$ реальная температура системы, $k-$ постоянная Больцмана.

В данной работе для компьютерного моделирования использовалась модель Изинга с кубической решеткой. Моделирование осуществлялось методом Монте-Карло с помощью алгоритма Метрополиса, дающим адекватные 
результаты для аналогичных систем [15]. Решетка имела линейные размеры $L \times L \times L$. Использовались периодические граничные условия. Температуры фазовых переходов определялись в рамках теории конечноразмерного скейлинга [15].

В исследуемой системе возможно, как ферромагнитное, так антиферромагнитное упорядочивание спинов. В связи с чем, необходимо введение двух параметров порядка. Ферромагнитный параметр порядка $m$ представляет собой простую сумму спинов в узлах решетки:

$$
m=\sum S_{i}
$$

Для описания антиферромагнитных фазовых переходов введем параметр порядка $m_{a}$, который вычисляется как шахматная намагниченность спинов. Антиферромагнитный параметр порядка равен разности магнитных моментов двух подрешеток спинов с четными и нечетными индексами:

$$
m_{a}=\sum_{\text {even }} S_{i}-\sum_{\text {odd }} S_{i} .
$$

Температуры фазовых переходов определялась с помощью куммулянтов Биндера четвертого порядка для ферромагнитного и антиферромагнитного параметров порядка [15]:

$$
U=1-\frac{\left\langle m^{4}\right\rangle}{3\left\langle m^{2}\right\rangle^{2}}, \quad U_{a}=1-\frac{\left\langle m_{a}^{4}\right\rangle}{3\left\langle m_{a}^{2}\right\rangle^{2}} .
$$

Угловыми скобками обозначено термодинамическое усреднение по различным состояниям.

Температура фазового перехода может быть найдена как точка пересечения куммулянтов Биндера для систем с различными линейными размерами $L$. Для нахождения температуры ферромагнитного перехода $T_{C}$ использовались куммулянты $U$, для определения температуры антиферромагнитного фазового перехода $T_{N}$ исследовалось поведение куммулянтов $U_{a}$.

\section{3. Результаты компьютерного моделирования}

Компьютерный эксперимент проводился для систем с линейными размерами от $L=20$ до $L=36$ с шагом $\Delta L=4$. Выполнялось $8 \cdot 10^{5}$ шагов Монте-Карло на спин. Использовались циклические граничные условия. Из данного вида граничных условий для антиферромагнитных систем вытекает требование рассмотрения при моделировании только систем с четными линейными размерами. Как показывают исследования для аналогичных задач выбранные линейные размеры позволяют определять величину температуры с точностью до второго знака после запятой. Увеличение размера системы существенно увеличивает время расчетов при изменении результатов моделирования лишь в третьем знаке после запятой. Тогда как данные реальных экспериментов такой точности на сегодняшний день отсутствуют. Параметр дальнодействия изменялся от $\sigma=1.6$ до $\sigma=1.9$ с шагом
$\Delta \sigma=0.1$. Из результатов, полученных для систем с эффектами дальнодействия, известно, что при $\sigma \leq 1.5$ наблюдается среднеполевое критическое поведение, а при $\sigma \geq 2.0$ эффекты дальнодействия несущественны. Относительная интенсивность дальнодействующих сил изменялась от $b=0.9$ до $b=1.4$ с шагом $\Delta b=0.1$. Напряженность магнитного поля изменялась от $h=0.0$ до $h=1.1$ с шагом $\Delta h=0.1$. При каждой наборе значений $(b, \sigma, h)$ определялась температура ферромагнитного $T_{C}$ или антиферромагнитного $T_{N}$ фазового перехода. На Рис. 1 приведена фазовая диаграмма системы для двух крайних рассматриваемых случаев скорости убывания дальнодействия с расстоянием при $\sigma=1.6$ и $\sigma=1.9$.

Как видно из Рис. 1 на фазовой диаграмме системы присутствует три фазы: парамагнитная (PM), ферромагнитная (FM) и антиферромагнитная (AFM). При понижении температуры система переходит из парамагнитной фазы в ферромагнитную или антиферромагнитную. Вид низкотемпературной фазы определяется относительной интенсивностью дальнодействующих антиферромагнитных сил. Как видно из Рис. 1 существует граничное значение относительной интенсивности $b_{0}$, выше которого реализуется антиферромагнитная фаза, а ниже ферромагнитная. Как показал компьютерный эксперимент, для всех рассматриваемых систем в отсутствии внешнего магнитного поля $b_{0}=0.9$.

Присутствие внешнего магнитного поля существенно меняет фазовую диаграмму системы. Прежде всего, наблюдается хорошо известный эффект размытия ферромагнитного фазового перехода во внешнем магнитном поле, в результате чего становится невозможным локализовать температуру Кюри. Кроме этого наблюдается снижение температуры антиферромагнитного фазового перехода, аналогично чистым

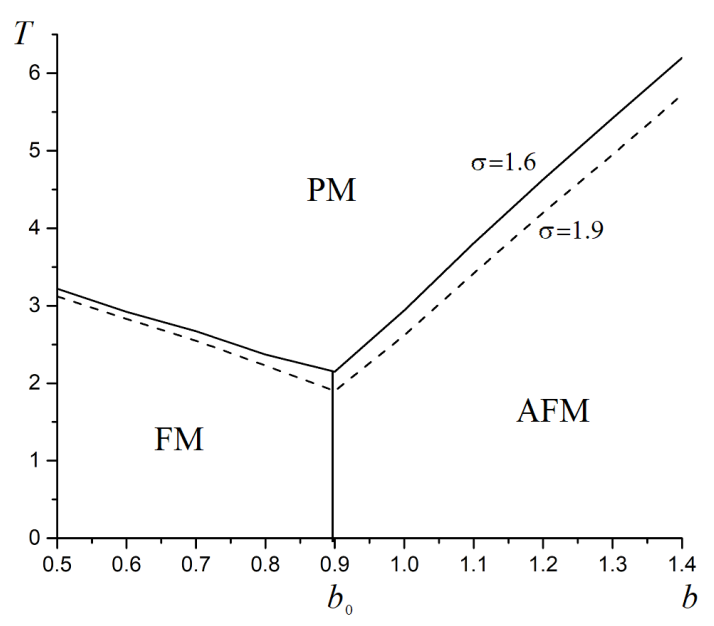

Рис. 1. Фазовая диаграмма системы для двух случаев $\sigma=1.6$ и $\sigma=1.9$. Сплошные линии соответствуют случаю $\sigma=1.6$, пунктирные $-\sigma=1.9$. FM - ферромагнитная фаза. AFM - антиферромагнитная фаза. PM - парамагнитная фаза.

Fig. 1. Phase diagram of system for two cases $\sigma=1.6$ and $\sigma=1$.9. Solid lines correspond to the case $\sigma=1.6$, dashed lines correspond to the case $\sigma=1.9$. FM - ferromagnetic phase. AFM - antiferromagnetic phase. $\mathrm{PM}-$ paramagnetic phase. 
антиферромагнитным системам. На Рис. 2 приведена зависимость температуры антиферромагнитного фазового перехода от напряженности внешнего магнитного поля для систем с параметром дальнодействия $\sigma=1.6$ и различной относительной интенсивностью дальнодействующих сил. Для систем с другими значениями параметра дальнодействия $\sigma$ зависимости имеют аналогичный вид.

Как и для обычных антиферромагнитных систем температура Нееля убывает по квадратичному закону, который может быть записан в виде:

$$
T=T_{0}\left(1-A(b, \sigma) h^{2}\right) .
$$

Коэффициент $A(b, \sigma)$ зависит от обоих параметров, определяющих дальнодействующие антиферромагнитные силы.

Еще один существенный эффект, вызванный присутствием внешнего магнитного поля, состоит в изменении пограничного значения $b_{0}$. Поэтому пограничное значение относительной эффективности дальнодействующих сил необходимо рассматривать как функцию от напряженности внешнего магнитного поля $b_{0}(h)$. Компьютерный эксперимент показал, что значения функции $b_{0}(h)$ не зависят от параметра дальнодействия $\sigma$. Зависимость $b_{0}$ от напряженности внешнего магнитного поля $h$ представлена на Рис. 3.

Из Рис. 3 можно сделать вывод о линейном росте пограничного значения относительной интенсивности дальнодействующих антиферромагнитных $b_{0}(h)$ сил c увеличением внешнего магнитного поля. Данная зависимость с высокой точностью описывается линейной функцией:

$$
b_{0}(h)=0.33 h+0.9
$$

В результате в системе с параметром $b>b_{0}(0)$, при нулевом внешнем магнитном поле, будет наблюдаться антиферромагнитная фаза. При помещении этой системы во внешнее магнитное поле может выполниться обратное неравенство $b<b_{0}(h)$, в результате чего будет

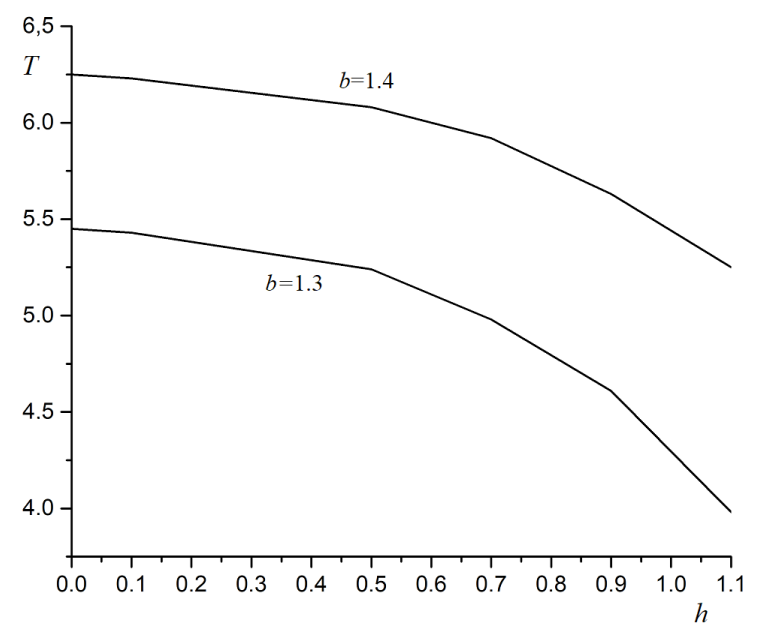

Pис. 2. Зависимость температуры антиферромагнитного фазового перехода от напряженности внешнего магнитного поля при $\sigma=1.6$.

Fig. 2. Dependence of the antiferromagnetic phase transition on magnetic field intensity for the case $\sigma=1.6$.



Рис. 3. Зависимость пограничного значения относительной эффективности дальнодействующих антиферромагнитных сил $b_{0}$ от напряженности внешнего магнитного поля $h$.

Fig. 3. Dependence of the boundary of intensity long-range antiferromagnetic force $b_{0}$ on the magnetic field intensity $h$.

наблюдаться ферромагнитная фаза. Экспериментально этот эффект будет выглядеть как фазовый переход из антиферромагнитной в ферромагнитную фазу под действием внешнего магнитного поля.

\section{4. Заключение}

Таким образом, в спиновых системах с конкуренцией между близкодействующим ферромагнитным взаимодействием и дальнодействующим антиферромагнитным взаимодействием параметры фазовых переходов зависят от напряженности внешнего магнитного поля. При относительной интенсивности дальнодействующих сил выше некоторого пограничного значения происходит антиферромагнитное упорядочение спинов, a ниже пограничного значения - ферромагнитное упорядочение спинов. Величина пограничного значения линейно растет с увеличением напряженности внешнего магнитного поля. В результате система может переходить из антиферромагнитной фазы в ферромагнитноую фазу. Данный фазовый переход наблюдался экспериментально в ряде веществ. Например, данный переход из антиферромагнитной фазы в ферромагнитную под действием внешнего магнитного поля описан в работе [16] для $\mathrm{Dy}_{2} \mathrm{Co}_{3} \mathrm{Al}_{9}$.

Эффект подавления антиферромагнитного порядка магнитным полем наблюдался экспериментально для сплава $\mathrm{Gd}_{5}\left(\mathrm{Si}_{x} \mathrm{Ge}_{1-x}\right)_{4}$ при $x \leq 0.1$ [17]. В отсутствие магнитного поля доминируют дальнодействующие антиферромагнитные силы. В данных сплавах антиферромагнитный фазовый переход происходит при температуре 127-134 К [18-26]. В магнитном поле при напряженности выше 14 кЭ происходит подавление антиферромагнитного дальнего порядка с появлением областей ферромагнитного упорядочения. При дальнейшем увеличении магнитного поля система полностью переходит в ферромагнитное состояние [19]. Аналогичные явления наблюдались экспериментально 
для этого сплава при $x=0,0.125,0.253,0.375,0.875$ при температурах ниже 215 К и магнитном поле выше 10 кЭ [27].

Сосуществование близкодействующих ферромагнитных сил и дальнодействующих антиферромагнитных также было обнаружено в монокристалле $\mathrm{Gd}_{5} \mathrm{Ge}_{4}[28,29]$. Причем в отсутствие магнитного поля при температурах ниже 230 К система находится в антиферромагнитной фазе. В магнитном поле, напряженность которого превышает 5 кЭ происходит подавление антиферромагнитных сил, в результате чего система переходит в ферромагнитную фазу.

Возможность изменять тип упорядочения спинов в системах с конкурирующими взаимодействиями делает их перспективными материалами для устройств спинтроники.

\section{Литература/References}

1. P. Kayser, M. J. Martinez-Lope, J. A. Alonso, M. Retuerto, M. Croft, A. Ignatov, M. T. Fernandez-Diaz. Inorg. Chem. 52, 11013 (2013). Crossref

2. X. Ou, Z. Li, F. Fan, H. Wang, H. Wu. Sci. Rep. 4, 7542 (2014). Crossref

3. J. Enkovaara, O. Heczko, A. Ayuela, R. M. Nieminen. Phys. Rev. B. 67, 212405 (2003). $\underline{\text { Crossref }}$

4. E. Luijten, H.W. J. Blote. Phys. Rev. B. 56, 8945 (1997). Crossref

5. S. V. Belim. JETP Letters. 77, 2, 112 (2003). Crossref

6. S. V. Belim. Herald of the Bauman Moscow State Tech. Univ., Nat. Sci. 1, 37 (2019). Crossref

7. E. Bayong, H.T. Diep. Phys. Rev. B. 59, 11919 (1999). Crossref

8. E. Luijten. Phys. Rev. E. 60, 7558 (1999). Crossref

9. S. V. Belim, I. B. Larionov, R. V. Soloneckiy. Phys. Metals Metallogr. 117, 1079 (2016). Crossref

10. W. Li, H. P. Kunkel, X.Z. Zhou, G. Williams, Y. Mukovskii, D. Shulyatev. J. Phys. Condens. Matter. 16, L109 (2004). Crossref

11. M. G. Pini, A. Rettori, P. Betti, J. S. Jiang, Y. Ji, S. G. E. te Velthuis, G.P. Felcher, S.D. Bader. J. Phys.: Condens. Matter. 19, 479003 (2007). Crossref
12. H.W. J. Blote, X.N. Wu. J. Phys. A. 23, L627 (1990). Crossref

13. A. A. Tarasenko, L. Jastrabik, F. Nieto, C. Uebing. Phys. Rev. B. 59, 8252 (1999). Crossref

14. X.-Z. Wang, J.S. Kim. Phys. Rev. Lett. 78, 413 (1997). Crossref 15. D. P. Landau, K. Binder. Phys. Rev. B. 17, 2328 (1978). Crossref

16. D.I. Gorbunov, M.S. Henriques, N. Qureshi, B. Ouladdiaf, C.S. Mejia, J. Gronemann, A.V. Andreev, V. Petricek, E. L. Green. Phys. Rev. Materials. 2, 084406 (2018). Crossref

17. F. Casanova, S. de Brion, A. Labarta, X. Batlle. J. Phys. D: Appl. Phys. 38, 3343 (2005). Crossref

18. V.K. Pecharsky, K.A. Gschneidner Jr. J. Alloys Compounds. 260, 98 (1997). Crossref

19. L. Morellon, J. Blasco, P.A. Algarabel, M.R. Ibarra. Phys. Rev. B. 62, 1022 (2000). Crossref

20. E. M. Levin, K.A. Gschneidner Jr., V.K. Pecharsky. Phys. Rev. B. 65, 214427 (2002). Crossref

21. C. Magen, L. Morellon, P. A. Algarabel, C. Marquina, M. R. Ibarra. J. Phys.: Condens. Matter, 15, 2389 (2003). Crossref

22. Y. Zhuo, R. Chahine, T. K. Bose. IEEE Trans. Magn. 39, 3358 (2003). $\underline{\text { Crossref }}$

23. V. Hardy, S. Majumdar, S. J. Crowe, M. R. Lees, D. McK. Paul, L. Herve, A. Maignan, S. Hebert, C. Martin, C. Yaicle, M. Hervieu, B. Raveau. Phys. Rev. B. 69, 020407 (R) (2004). Crossref

24. F. Casanova, A. Labarta, X. Batlle, J. Marcos, L. Manosa, A. Planes, S. de Brion. Phys. Rev. B. 69, 104416 (2004). Crossref

25. H. Tang, V.K. Pecharsky, K.A. Gschneidner Jr., A. O. Pecharsky. Phys. Rev. B. 69, 064410 (2004). Crossref

26. V. K. Pecharsky, K. A. Gschneidner Jr. Appl. Phys. Lett. 70, 3299 (1997). Crossref

27. Z.W. Ouyang. J. of Appl. Phys. 108, 033907 (2010). Crossref

28. Z.W. Ouyang, V.K. Pecharsky, K.A. Gschneidner Jr., D. L. Schlagel, T. A. Lograsso. Phys. Rev. B. 74, 094404 (2006). Crossref

29. N. Perez, F. Casanova, F. Bartolome, L. M. Garcia, A. Labarta, X. Batlle. Phys. Rev. B. 83, 184411 (2011). Crossref 